\title{
A case report of glycogen storage disorder in an adult
}

\author{
Authors: Kyaw Linn Su Khin, ${ }^{\mathrm{A}}$ Sara Mahgoub, ${ }^{\mathrm{A}}$ Thea Haldane ${ }^{\mathrm{A}}$ and Hwee Phen Teh ${ }^{\mathrm{A}}$
}

\section{Background}

Glycogen storage disorder (GSD) is a rare cause of chronic liver disease especially in the adult population comprising of many different subtypes. This results from absence of enzymes which are involved in converting glycogen compounds to glucose, and this subsequently results in accumulation of glycogen in tissues. Among the various subtypes, GSD III is one of the most common subtypes, inherited as an autosomal recessive disease accounting for 2.3 in 100,000 children in the United States per year.

\section{Case presentation}

We present a case of 25-year-old man with 3-month history of jaundice, generalised malaise, weight loss, chronic abdominal pain and inability to concentrate. He was normally fit, drinking socially and denied taking any medications including recreational drugs. There was no significant family history of liver disease.

On examination, he was pale, jaundiced with noticeable full cheeks, with below-the-average height. There were many bruises and purple striae. Liver was not palpable; spleen was just palpable on deep palpation. There was no ascites and no other stigmata of chronic liver disease nor proximal myopathy. Random blood sugar was $4.6 \mathrm{mmol} / \mathrm{L}$.

His initial liver function test showed bilirubin of $101 \mu \mathrm{mol} / \mathrm{L}$ with normal enzymes, albumin $25 \mathrm{~g} / \mathrm{L}$, clotting test showed prothrombin 22 seconds and international normalised ratio (INR) of 1.8. Full blood count showed pancytopenia with normal reticulocyte count and raised lactate dehydrogenase (LDH). Blood film showed left-shifted neutropenia with thrombocytopenia with red cell anisopoikilocytosis with occasional target cells. There was no fragmentation. Bone marrow biopsy was unremarkable. Alpha fetoprotein was slightly raised while carcinoembryonic antigen, CA199, CA153 and human chorionic gonadotrophin were normal. All autoantibody screens were normal with negative viral screening. Total iron binding capacity and serum iron were raised but ferritin and copper were normal.

Ultrasound showed splenomegaly with a small low-

attenuation liver lesion. Hence, he had liver magnetic resonance imaging and computed tomography of the liver triple phase to further evaluate the lesion; this confirmed cirrhosis with dysplastic nodules and splenomegaly. Liver biopsy was performed afterwards and revealed significant glycogen load in hepatocytes on special stains but there was no evidence of increased iron, amyloid deposition, copper-associated protein nor evidence of alpha 1 anti-trypsin deficiency.

He was then referred to liver team for further monitoring and management.

\section{Discussion}

GSD III is a very rare cause of jaundice and median age of presentation is within the first year of life. The most common presentations include full cheeks, hypertriglyceridemia, hypoglycaemia, immunodeficiency, intellectual disability, short stature and myopathy. Typically, GSD should be suspected when three main clinical features are present: hepatomegaly, hypoglycaemia, and elevated liver enzymes and creatinine kinase. This condition is diagnosed by histology and genetic testing.

Unfortunately, there is no specific treatment for this condition. The aim of the treatment is to alleviate symptoms by maintaining euglycaemia. This can be obtained by frequent meals high in carbohydrates. There is risk of malignant transformation and overt liver failure.

\section{Conclusion}

In conclusion, although GSD mostly presents in childhood, there can be rare exceptions to this. Timely diagnosis, interval monitoring and timely management can prevent serious complications like overt liver failure and hepatocellular carcinoma.

\section{Conflicts of interest}

None declared. 